\title{
Armazenamento caseiro de medicamentos por usuários restritos ao domicílio da área de abrangência do Centro de Saúde Jaqueline I, Regional Norte, Município de Belo Horizonte, Brasil.
}

\author{
Household storage of medicines by restricted users at home in the \\ area covered by the Jaqueline I Health Center, Northern Region, \\ Municipality of Belo Horizonte, Brazil
}

Recebido em: 16/02/2017

Aceito em: $21 / 08 / 2017$
Lia Silva de CASTILHO' ${ }^{1}$; Gilberto ROCHA FILHO'

Andréa Clemente PALMIER ${ }^{1}$

${ }^{1}$ Universidade Federal de Minas Gerais. Avenida Presidente Antônio Carlos, 6627, Bairro Pampulha, CEP 31270-010, Belo Horizonte, MG, Brasil. ${ }_{2}^{2}$ Prefeitura Municipal de Belo Horizonte. Centro de Saúde Jaqueline II.

R. João Pereira Lima, 50, Bairro Jaqueline, CEP 31748-147, Belo Horizonte, MG, Brasil.E-mail: liasc@ufmg.br

\section{ABSTRACT}

This work aimed to investigate the homemade stock of medicines of homebound persons of a health center in Belo Horizonte, Minas Gerais, Brazil. Socio-demographic and economic variables; type of stored drug, its validity and the storage location was collected through an applied questionnaire to the patients. Descriptive analysis was performed. Forty-three patients, living in 42 houses were visited and was possible to observe a predominance of women (67.44\%), old people (72.10\%) and low family income (67,42\% earned up to 3 minimum wages). The most commonly found medications were related to the nervous and cardiovascular systems, metabolism and alimentary tract diseases. Only one home (2.4\%) did not have stocked medicine.Among the total of 14,385 doses/units found, $12,346(85.8 \%)$ were for continuous treatment and 2,966 (20.6\%) were considered unsuitable for consumption. The found medicines were consistent with the chronic degenerative pathologies presented. The found units/dose demonstrates a difference between the amount of product dispensed and actual use by the patient. Therefore, to control the stocked drugs can bring benefit to the patient, economy for the health service and a reduction of the disposal of waste medicines.

Keywords: drug storage; homebound persons; waste management; pharmaceutical services

\section{RESUMO}

Esse estudo investigou o estoque caseiro de medicamentos de todos os indivíduos restritos ao domicílio de um Centro de Saúde em Belo Horizonte, Minas Gerais. As variáveis sociodemográficas e econômicas dos pacientes; o tipo de medicamento, sua validade e o local de armazenamento foram coletados por meio de um questionário. Uma análise descritiva foi realizada. Foram visitados 43 pacientes em 42 casas, com predominância de mulheres (67,44\%), idosos (72,10\%), com baixa renda familiar (67,42\% recebiam até 3 salários mínimos). Os medicamentos mais encontrados foram para os sistemas nervoso e cardiovascular, para o metabolismo e trato alimentar. Apenas um (2,4\%) lar não apresentava medicamento estocado. Do total de 14.385 doses/unidades encontradas, $12.346(85,8 \%)$ eram de uso contínuo e $2.966(20,6 \%)$ foram consideradas como inapropriadas para o consumo. O estoque de medicamentos en- 
contrado coaduna com as patologias crônico-degenerativas apresentadas. O volume de unidades/doses encontradasmostrou uma diferença entre a quantidade de medicamento dispensada e a real utilização pelo paciente. Uma estratégia de controle deste volume de medicamentos pode trazer benefícios para o paciente, economia para o serviço e controle do descarte de medicamentos inapropriados para uso.

Palavras-chave: armazenagem de medicamentos; pacientes domiciliares; gerenciamento de resíduos; assistência farmacêutica

\section{INTRODUÇÃO}

A armazenagem de medicamentos no domicílio é uma prática comum em todo o mundo, variando de percentuais inferiores a $40 \%$ na Etiópia (1) e em Uganda (2), podendo atingir 90\% em alguns países (3-6). No Brasil, a taxa daqueles que armazenam medicamentos é superior a $90 \%$, relatada por pesquisas que fizeram busca ativa nos domicílios (7-12). A justificativa para esta prática é a de guardar para futuras necessidades (2). O estoque caseiro de medicamentos pode facilitar a automedicação $(2,13)$ e interferir na efetividade do medicamento, nos casos em que o mesmo não é adequadamente guardado (12).

Entre idosos, especialmente aqueles restritos ao domićlio, é esperado que a quantidade de medicamentos estocados seja maior do que nos demais casos, considerando que estes indivíduos apresentam uma maior prevalência de doenças crônico-degenerativas (14), sendo comum o fenômeno da polifarmácia (ou polimedicação, que refere-se ao consumo de 4 medicamentos ou mais simultaneamente) nesta faixa etária (15). Consequentemente, os tipos de medicamentos estocados são diferentes daqueles armazenados pela população mais jovem, pois é esperada uma maior quantidade de medicamentos de uso contínuo (16).

Em um estudo de prevalência de idosos restritos aos domicílios na região metropolitana de Belo Horizonte, MG, os autores, após entrevistarem 275 idosos adscritos ao Programa Saúde da Família do Centro de Saúde Ventosa, identificaram que $22,4 \%$ eram restritos aos domicílios (17). Como esses idosos frágeis consomem muitos medicamentos, o Agente Comunitário de Saúde (ACS) constitui-se um profissional indispensável para o controle do estoque caseiro de medicamentos, podendo colaborar extensivamente para o seu uso racional. $\mathrm{Na}$ identificação dos pacientes restritos e agendamento das visitas dessa pesquisa, fez-se necessária a colaboração desses profissionais, que possuem íntimo conhecimento das famílias a serem visitadas (12).

Em 2015, o Brasil dispendeu R\$2.799.856.370,38 para Assistência Farmacêutica. Belo Horizonte rece- beu, por meio de transferência direta do Fundo Nacional de Saúde (FNS), via Secretaria Estadual, R\$ 59.398.183,60; e para o Programa Farmácia Popular, transferência direta do FNS para o município, R\$ 59.725.297,32 (18) Como os recursos financeiros utilizados para assistência farmacêutica no país são elevados, existindo a possibilidade de ocorrência de elevados estoques domiciliares de medicamentos e de perdas desses, o armazenamento caseiro de medicamentos pode se configurar em um problema para a sociedade e para uma eficiente política de distribuição de medicamentos. Assim, este estudo teve como objetivo investigar a estocagem de medicamentos de pacientes com restrição domiciliar da área de abrangência do Centro de Saúde (CS) Jaqueline I, regional Norte, Município de Belo Horizonte, assistidos por cinco Equipes de Saúde da Família e atendidos pelas Equipes de Saúde Bucal 1 e 2 (ESB 1 e 2).

\section{MÉTODOS}

Considerações Éticas. Este estudo foi aprovado pelo COEP-UFMG sob o número 37394514.2.0000.5149 em 09/02/2015.

Delineamento do Estudo. Este é um estudo transversal realizado na área de abrangência do Centro de Saúde Jaqueline I, localizado na região Norte no Município de Belo Horizonte, Minas Gerais. A característica do trabalho é de um censo, pois todos os pacientes restritos ao domicílio desta área foram visitados. O Centro de Saúde Jaqueline I atende uma população de aproximadamente 14.800 pessoas (cadastro oficial da Prefeitura de Belo Horizonte), considerada como de elevado e muito elevado risco social.

Processo de calibração. Um questionário estruturado foi aplicado por apenas um pesquisador treinado, sendo submetido previamente a um teste e reteste em 10 lares de usuários não restritos, para averiguação da reprodutibilidade do questionário com $100 \%$ de sucesso. Esse pesquisador foi o único responsável também pela averiguação da estocagem dos medicamentos durante as visitas. 
Instrumento de Coleta de Dados. A elaboração do questionário baseou-se em estudos anteriores $(10,19)$ e continha questões sobre: idade, gênero, doenças e quadros neurológicos relacionados à restrição domiciliar, renda por domicílio, presença de crianças coabitando com o paciente, escolaridade do cuidador, o tipo, a quantidade, a forma de aquisição e a prescrição de medicamentos presentes nos domicílios, a data de vencimento e a forma como estavam armazenados (exposição à luz/calor/umidade e ao alcance das crianças).

Procedimento de coleta de dados. As visitas domiciliares foram agendadas pelos ACS após prévio contato com os familiares e/ou responsáveis, de modo a não prejudicar as atividades relativas aos cuidados diários desses pacientes restritos.

Análise dos dados. Como se tratou de um censo, os dados foram tabulados no Microsoft Excel 2010 para realizar a análise descritiva em percentuais. Para isso, os medicamentos foram classificados inicialmente de acordo com a Relação Nacional dos Medicamentos Essenciais (RENAME) (20) e, em seguida, para identificação das classes terapêuticas, foram classificados por meio do
Anatomical Therapeutic Chemical (ATC) (21). Os dados foram dispostos, posteriormente, em função das faixas etárias determinadas de acordo com a classificação do Instituto Brasileiro de Estatística (IBGE) e em função do gênero (22).

\section{RESULTADOS E DISCUSSÃO}

Dos 48 pacientes inicialmente identificados nos prontuários eletrônicos, sete foram excluídos por motivo de mudança de bairro (1), falecimentos (4), recolhimento ao sistema penitenciário (1) e internação hospitalar (1). Duas novas pacientes foram acrescentadas após se mudarem para a área de abrangência. Com isso foram visitados, ao todo, 43 pacientes em 42 residências: em um dos lares visitados, residiam dois pacientes restritos. A faixa etária prevalente foi a de 60 e 79 anos, 21 pacientes $(48,84 \%)$, seguidos por $12(27,91 \%)$ pacientes até 59 anos e por $10(23,26 \%)$ pacientes na faixa etária igual ou superior a 80 anos. As idades tiveram variação de 11 anos até 95 anos, média de 63,74 anos, mediana igual a 68 anos; sendo $29(67,44 \%)$ indivíduos eram do sexo feminino (Tabela 1).

Tabela 1. Caracterização dos pacientes restritos visitados e seus cuidadores - principais dados, Centro de Saúde Jaqueline I, Belo Horizonte, Minas Gerais, 2015

\begin{tabular}{|c|c|c|}
\hline Idade & $\mathrm{n}$ & $\%$ \\
\hline$<59$ anos & 12 & 27,90 \\
\hline $60-79$ anos & 21 & 48,84 \\
\hline$>80$ anos & 10 & 23,26 \\
\hline Gênero & $\mathrm{n}$ & $\%$ \\
\hline Feminino & 29 & 67,44 \\
\hline Masculino & 14 & 32,56 \\
\hline Renda Familiar Mensal & $\mathrm{n}$ & $\%$ \\
\hline 1 SM & 3 & 6,97 \\
\hline $1-2 S M$ & 14 & 32,55 \\
\hline $2-3 \mathrm{SM}$ & 12 & 27,90 \\
\hline $3-5$ SM & 9 & 20,93 \\
\hline$>5 \mathrm{SM}$ & 2 & 4,65 \\
\hline Não respondeu & 3 & 6,97 \\
\hline Bolsa Família & $\mathrm{n}$ & $\%$ \\
\hline Sim & 10 & 23,25 \\
\hline Não & 33 & 76,74 \\
\hline Ter Cuidador & n & $\%$ \\
\hline Não possui & 2 & 4,65 \\
\hline Cuidador contratado & 6 & 13,95 \\
\hline Cuidador familiar & 35 & 81,40 \\
\hline
\end{tabular}




\section{loctarma}

Continuação da Tabela 1

\begin{tabular}{|c|c|c|}
\hline Escolaridade do Cuidador & n & $\%$ \\
\hline Sem escolaridade & 2 & 4,65 \\
\hline Fundamental Incompleto & 23 & 53,48 \\
\hline Fundamental Completo & 3 & 6,97 \\
\hline Ensino Médio Incompleto & 3 & 6,97 \\
\hline Ensino Médio Completo & 10 & 23,25 \\
\hline Superior completo & 1 & 2,32 \\
\hline Não respondeu & 1 & 2,32 \\
\hline Crianças (lares com) & n & $\%$ \\
\hline Sim & 23 & 53,48 \\
\hline Não & 20 & 46,51 \\
\hline Paciente é capaz de sair de casa sozinho? & $\mathrm{n}$ & $\%$ \\
\hline Sim, amparado & 3 & 6,97 \\
\hline Não & 40 & 93,03 \\
\hline Nivel de restrição & n & $\%$ \\
\hline Restrito a vizinhança & 3 & 6,97 \\
\hline Restrito à residência & 24 & 55,81 \\
\hline Restrito ao leito (acamado) & 16 & 37,22 \\
\hline Fatores dificultadores & n & $\%$ \\
\hline Casa não adaptada (degraus/sem corrimãos/becos estreitos) & 28 & 65,11 \\
\hline Não respondeu/não sabe & 15 & 34,89 \\
\hline Uso contínuo de medicamentos & $\mathrm{n}$ & $\%$ \\
\hline Sim & 42 & 97,67 \\
\hline Não & 1 & 2,33 \\
\hline Onde adquiriu medicamentos & $\mathrm{n}$ & $\%$ \\
\hline Centro de Saúde & 23 & 53,49 \\
\hline Farmácia particular & 2 & 4,65 \\
\hline Ambos (Centro de saúde + farmácia particular) & 18 & 41,86 \\
\hline Recebeu orientações sobre uso do medicamento & $\mathrm{n}$ & $\%$ \\
\hline Sim & 36 & 83,72 \\
\hline Não & 7 & 16,28 \\
\hline Auto Percepção de Saúde & n & $\%$ \\
\hline Muito bom/bom & 19 & 44,18 \\
\hline Moderado & 20 & 46,51 \\
\hline Ruim/Muito Ruim & 4 & 9,30 \\
\hline Quem respondeu & $\mathrm{n}$ & $\%$ \\
\hline Paciente & 6 & 13,95 \\
\hline Cuidador & 27 & 62,79 \\
\hline Ambos (paciente + cuidador) & 10 & 23,26 \\
\hline
\end{tabular}

Em relação à renda familiar, em $28(66,67 \%)$ residências foi declarado receber de um a três salários mínimos e em 10 residências $(23,80 \%)$ houve relato de participação no Programa-Bolsa Família (Tabela 1).
Do total de pacientes, $81,40 \%$, estavam sob os cuidados de familiares e apenas 6 tinham um cuidador contratado. Em relação à escolaridade dos cuidadores, 27 $(64,28 \%)$ possuíam somente o ensino fundamental com- 
pleto (Tabela 1). Em 10 (23,25\%) domicílios visitados não havia crianças que passavam o dia sob a supervisão desses cuidadores (Tabela 1).

Do total de pacientes, $97,67 \%$ declararam utilizer medicamentos de uso contínuo e $23(53,48 \%)$ adquiriram os medicamentos exclusivamente no Centro de Saúde (Tabela 1). Trinta e seis $(83,72 \%)$ indivíduos declararam que receberam orientações sobre o uso correto de medicamentos e que esse procedimento foi bem realizado pelos serviços de saúde (Tabela 1).

Motivos para a Restrição domiciliar. Foram relatados 21 diferentes motivos para a restrição domiciliar, sendo que o mais comum foi o Acidente Vásculo-Encefálico (AVE), responsável por 14 casos (Tabela 2). Os dez pacientes acometidos por AVE pertenciam predominantemente à faixa etária de 60 a 79 anos. Dentre as patologias adicionais relatadas pelos entrevistados, a de maior prevalência foi a Hipertensão Arterial Sistêmica (HAS) (Tabela 3) que afeta sete pacientes do sexo masculino e 20 do sexo feminino, com idade média de 69,55 anos.
Medicamentos. Foram encontrados 152 diferentes medicamentos nas residências dos pacientes visitados, dos quais $117(76,97 \%)$ eram para uso contínuo, com uma frequência de 404 vezes e 97 (63,81\%) pertenciam à RENAME (20). Segundo a classificação ATC (21), $25 \%$ eram para o sistema nervoso, $18,42 \%$ para metabolismo e trato alimentar e $15,13 \%$ para doenças do sistema cardiovascular.

Quando foi analisada a frequência com que esses medicamentos foram encontrados, foi possível observar que os 38 medicamentos para sistema nervoso foram encontrados 110 vezes $(27,22 \%)$, os 23 medicamentos para sistema cardiovascular 101 vezes $(25 \%)$ e, os 28 medicamentos para metabolismo e trato alimentar 82 vezes $(20,29 \%)$ (Tabela 4). O gênero feminino da faixa etária maior do que 60 anos concentrou a maior frequência de medicamentos encontrados em residências (225 ocorrências ou 55,69\%). A média de diferentes tipos de medicamentos por paciente foi de 9,39 (404/43). A média até 59 anos foi de 9,16 medicamentos por paciente e a média para os indivíduos com idades acima de 60 anos foi de 9,48 medicamentos.

Tabela 2. Motivos para a restrição domiciliar, pacientes do CS Jaqueline 1, visitados pela Equipe de Saúde Bucal 1, Belo Horizonte, Minas Gerais, Brasil, 2015

\begin{tabular}{|l|r|r|}
\hline \multicolumn{1}{|c|}{ Motivo para a restrição } & n & \multicolumn{1}{|c|}{$\%$} \\
\hline 1. Acidente Vásculo-Encefálico & 14 & 26,41 \\
\hline 2. Dificuldade de raciocínio/déficit cognitivo/demência & 6 & 11,32 \\
\hline 3. Mal de Alzheimer & 5 & 9,43 \\
\hline 4. Paralisia cerebral & 4 & 7,54 \\
\hline 5. Sequelas de poliomielite / indivíduo cadeirante & 3 & 5,66 \\
\hline 6. Depressão & 2 & 3,77 \\
\hline 7. Mal de Parkinson & 2 & 3,77 \\
\hline 8. Fratura/lesão medular & 2 & 3,77 \\
\hline 9. Acidente de motocicleta com múltiplas fraturas/ perdas ósseas / trauma medular & 2 & 3,77 \\
\hline 10. Hidrocefalia cerebral ou hidrocele & 2 & 3,77 \\
\hline 11. Infarto Agudo do Miocárdio & 1 & 1,89 \\
\hline 12. Artrose/artrite & 1 & 1,89 \\
\hline 13. Tumor de próstata & 1 & 1,89 \\
\hline 14. Câncer de cérebro & 1 & 1,89 \\
\hline 15. Queda com fratura de fêmur & 1 & 1,89 \\
\hline 16. Meningite bacteriana & 1 & 1,89 \\
\hline 17. Edema pulmonar/crise hipertensiva & 1 & 1,89 \\
\hline 18. Diminuição da acuidade visual/auditiva & 1 & 1,89 \\
\hline 19. Quedas frequentes & 1 & 1,89 \\
\hline 20. Coréia de Huntington & 1 & 1,89 \\
\hline 21. Sequelas de sífilis terciária/fraqueza & 1 & 1,89 \\
\hline Frequência total & $\mathbf{1 0}$ & $\mathbf{1 0 0}$ \\
\hline Média/paciente (53/43) & $\mathbf{1 , 2 3}$ & \\
\hline
\end{tabular}

*Alguns pacientes apresentam mais de um motivo para a restrição domiciliary. \%: representa o percentual de ocorrência da patologia/ frequência total. 
O ácido acetilsalicílíco (100 mg) foi o medicamento mais comumente encontrado (20 domicílios), seguido pela sinvastatina, encontrada em 18 lares. O omeprazol e o enalapril foram encontrados em 16 casas e o clonazepan e a dipirona em 12 . No presente estudo, ácido acetilsalicílico (devido à concentração de $100 \mathrm{mg}$ mais comumente encontrada) foi categorizado em medicamento para o sistema sanguíneo e órgãos hematopoiéticos, o enalapril e a sinvastatina em medicamentos para o sistema cardiovascular e o omeprazol foi categorizado em medicamento para o metabolismo e trato alimentar.

Analgésicos como dipirona e paracetamol e o benzodiazepínico clonazepan foram categorizados em medicamentos para o sistema nervoso, como classificado pela ATC (21). Foi observado um pequeno estoque de antimicrobianos de uso sistêmico: apenas 8 medicamentos utilizados por 12 pacientes, sendo que 1 não se encontrava listado na RENAME (20).
Nas visitas domiciliares, o número total de unidades/doses de medicamentos foi de 14.385 unidades (cápsulas, comprimidos, drágeas, xaropes, supositórios, tabletes, saches, diskus, pomadas, frascos e carpules). Medicamentos fora do prazo de validade e sem identificação totalizaram $2.966(20,62 \%)$ unidades/doses. Foram apresentadas as respectivas receitas médicas ou odontológicas para $13.723(95,40 \%)$ unidades/doses (Tabela 5).

Estoque dos medicamentos. Os medicamentos encontrados estavam armazenados adequadamente em 12 (28,57\%) domicílios. Nas demais moradias os medicamentos estavam sob exposição da luz, do calor, da umidade e ao alcance das crianças. Não foi possível determinar corretamente os cômodos que servem para guardar os medicamentos devido à disposição destes nas moradias investigadas. As residências são muito pequenas, sendo que às vezes um cômodo serve de sala, quarto e local de refeições. De qualquer modo, o armazenamento nos quartos/salas foi frequente em $28(66,66 \%)$ casos.

Tabela 3. Patologias adicionais aos motivos de restrição domiciliar dos pacientes restritos visitados, CS Jaqueline I, Belo Horizonte, Minas Gerais, Brasil, 2015

\begin{tabular}{|l|r|r|}
\hline \multicolumn{1}{|c|}{ Patologias adicionais ao motivo de restrição } & Total & $\%$ \\
\hline 1. Hipertensão Arterial Sistêmica & 27 & 28,73 \\
\hline 2. Diabetes & 12 & 12,76 \\
\hline 3. Depressão & 9 & 9,57 \\
\hline 4. Hipercolesterolemia & 6 & 6,38 \\
\hline 5. Glaucoma & 4 & 4,26 \\
\hline 6. Insuficiência renal/doença renal crônica & 3 & 3,20 \\
\hline 7. Hipotireoidismo & 3 & 3,20 \\
\hline 8. Catarata & 3 & 3,20 \\
\hline 9. Insuficiência cardíaca & 3 & 3,20 \\
\hline 10. Convulsões & 3 & 3,20 \\
\hline 11. Acidente Vasculo-Encefálico & 3 & 3,20 \\
\hline 12. Insônia & 2 & 2,13 \\
\hline 13. Fibrilação Atrial Crônica & 2 & 2,13 \\
\hline 14. Arritmias Cardíacas & 1 & 1,06 \\
\hline 15. Osteoporose & 1 & 1,06 \\
\hline 16. Tumor de próstata & 1 & 1,06 \\
\hline 17. Refluxo Gastro-esofágico & 1 & 1,06 \\
\hline 18. Anemia & 1 & 1,06 \\
\hline 19. Neuropatia medular & 1 & 1,06 \\
\hline 20. Infecções recorrentes & 1 & 1,06 \\
\hline 21. Doença de Chagas & 1 & 1,06 \\
\hline
\end{tabular}


Continuação da Tabela 3

\begin{tabular}{|c|c|c|}
\hline Patologias adicionais ao motivo de restrição & Total & $\%$ \\
\hline 22. Problemas de coluna & 1 & 1,06 \\
\hline 23. Hérnia de disco & 1 & 1,06 \\
\hline 24. Úlcera gástrica & 1 & 1,06 \\
\hline 25. Apnéia respiratória & 1 & 1,06 \\
\hline 26. Constipação intestinal & 1 & 1,06 \\
\hline 27. Ansiedade & 1 & 1,06 \\
\hline Frequência total ${ }^{*}$ & 94 & 100 \\
\hline Média/paciente(94/43) & 2,18 & \\
\hline
\end{tabular}

Tabela 4. Medicamentos encontrados nas residências dos pacientes restritos, segundo classificação ATC/OMS, CS Jaqueline I, Belo Horizonte, Minas Gerais, 2015

\begin{tabular}{|l|r|r|}
\hline \multicolumn{1}{|c|}{ Medicamentos por tipo de ação /ATC/OMS } & n & $\%$ \\
\hline Sistema Nervoso Central & 38 & 25,00 \\
\hline Metabolismo e trato alimentar & 28 & 18,42 \\
\hline Sistema cardiovascular & 23 & 15,13 \\
\hline Outros $\quad$ Frequência de medicamentos encontrados/ATC/OMS & 63 & 41,45 \\
\hline Total & $\mathbf{1 5 2}$ & $\mathbf{1 0 0}$ \\
\hline \multicolumn{1}{|c|}{ Sistema Nervoso Central } & $\mathbf{n}$ & $\%$ \\
\hline Sistema Cardiovascular & 110 & 27,22 \\
\hline Metabolismo e trato alimentar & 101 & 25,00 \\
\hline Outros & 82 & 20,29 \\
\hline Total & 111 & 27,49 \\
\hline
\end{tabular}

Foi observado um maior percentual de mulheres com idade acima de 60 anos. A média e mediana das idades foram menores do que as apresentadas por estudo anterior (23), porém são semelhantes aos resultados apresentados para a cidade de Belo Horizonte (média de 69,7 anos e predominância do gênero feminino) (24). A "feminização do envelhecimento" é um fenômeno observado não só no Brasil $(19,25-27)$, mas em todo o mundo, podendo ser explicado por uma vida menos exposta a riscos e com os efeitos protetores hormonais, diferenças metabólicas e genéticas; ou seja, é maior a frequência de doenças crônico-degenerativas entre mulheres idosas (28) e, consequentemente, há um maior uso de medicamentos para o controle dessas doenças. Além disso, mulheres idosas apresentam uma maior tendência a apresentarem deficiências na vida tardia. Nos Estados Unidos da América, porém, com o passar das décadas, foi observada uma diminuição das diferenças na expectativa de vida entre os gêneros (29).
O baixo poder econômico e o baixo nível de escolaridade deste grupo populacional corroboram os resultados apurados em relação à renda mensal dos domicílios, à dependência da farmácia do centro de Saúde para conseguir medicamentos (em mais da metade dos casos), à baixa escolaridade dos cuidadores, à incorreta armazenagem dos medicamentos, ao uso de medicamentos da lista da RENAME e pelo pequeno tamanho dos lares. Acrescenta-se a isso, o fato dos cuidadores também serem responsáveis pela guarda de crianças.

Os medicamentos dispensados no Centro de Saúde fazem parte da RENAME (20). Esses medicamentos correspondem a $64 \%$ do total de tipos de medicamentos, percentual bem maior ao encontrado anteriormente no Sul do Brasil, sendo basicamente para o tratamento de doenças crônico-degenerativas. Este resultado difere do estudo anterior que encontrou dentre os medicamentos essenciais a maior parte das indicações para uso agudo (8), onde encontraram uma associação entre o uso de medicamentos da RENAME e baixa renda dos pacientes. 
Baixa escolaridade e baixa renda são variáveis que afetam negativamente a efetiva orientação terapêutica (por parte daquele que dispensa o medicamento), a apreensão das informações (por parte do usuário) e o acesso aos fármacos (19). Estes fatores também influenciam negativamente a forma como o armazenamento de medicamentos é feito em casa. Por isso os profissionais de saúde devem se esforçar em empregar uma linguagem acessível ao usuário (12), levando em consideração o seu nível sócio-econômico-cultural (19). De fato, chefes de família que apresentavam menor grau de instrução, apresentavam maior risco de terem em casa medicamentos vencidos (6).

Tabela 5. Principais resultados encontrados durante a avaliação de unidades/doses de medicamentos nas visitas domiciliares aos pacientes restritos do Centro de saúde Jaqueline I, Belo Horizonte, MG, 2015

\begin{tabular}{|l|r|r|}
\hline \multicolumn{1}{|c|}{ Unidades/doses de medicamentos } & \multicolumn{1}{|c|}{$\mathbf{n}$} \\
\hline Total doses/unidades de medicamentos encontradas & 14385 \\
\hline Medicamentos válidos & 100 \\
\hline Medicamentos inservíveis & 11419 & 79,38 \\
\hline Medicamentos sem identificação & 2966 & 20,62 \\
\hline Medicamentos com identificação & 84 & 0,58 \\
\hline Medicamentos de uso contínuo & 14301 & 99,42 \\
\hline Medicamentos de uso eventual & 12350 & 85,86 \\
\hline Medicamentos sem identificação & 1951 & 13,56 \\
\hline Medicamentos com receita médico-odontológica & 84 & 0,58 \\
\hline Medicamentos sem receita médico-odontológica & 13712 & 95,32 \\
\hline Medicamentos sem identificação & 589 \\
\hline Medicamentos para Sistema Cardiovascular & 4,10 \\
\hline Medicamentos para Sistema Nervoso & 84 & 0,58 \\
\hline Medicamentos para Metabolismo/trato alimentar & 38,06 \\
\hline Demais medicamentos & 5475 \\
\hline
\end{tabular}

$\mathrm{n}$ representa o número de unidades/doses de medicamentos encontrados. Média de unidades medicamentos/paciente restrito (14385/43): 334,53

Do total de doses/unidades de medicamentos encontradas, cerca de $96 \%$ dos medicamentos possuíam a correspondente prescrição, indicando que o fenômeno da automedicação é pequeno entre esta população. Curiosamente, no Iraque, famílias cujos indivíduos possuíam grau universitário se automedicavam e armazenavam maior número de medicamentos em casa com maior frequência do que as famílias que possuíam menor grau de escolaridade (6).

Quanto ao número de domicílios que apresentavam o estoque caseiro de medicamentos, os achados nessa investigação têm resultados semelhantes aos da literatura pesquisada no Brasil $(7,8,10-12)$, e no exterior $(3,5,6)$.

Quando apenas os antimicrobianos são enfocados, os achados são inferiores aos encontrados na Rússia (4), no Iraque (6), em Uganda (2), na Etiópia (1) e no Brasil $(10,19)$. Este fato pode ser resultado da política de controle da sua venda e dispensação introduzida no Brasil a partir de 2011 (30).
O baixo poder aquisitivo leva o paciente a depender da dispensação farmacêutica do Centro de Saúde. Assim, o acesso ao medicamento vai depender das políticas públicas, de acordo com os países estudados. Depender do poder público para conseguir os medicamentos pode ser encarado como um fator positivo no controle do uso dos mesmos.

A dispensação só é realizada no centro de saúde se o paciente apresentar a receita médica ou odontológica. Com isso, os serviços passam a ter um maior controle do que está sendo utilizado. A quantidade de indivíduos que retiram o medicamento no Centro de Saúde foi maior do que as encontradas em estudos prévios $(11,12)$. Esse resultado, somado ao maior número de medicamentos que consta da lista RENAME, corrobora com estudos onde a presença no estoque caseiro de medicamentos essencial está é associada positivamente com a aquisição em centros de saúde (8). Outrossim, quanto maior é o poder aquisitivo das 
famílias maior é a possibilidade de aquisição dos medicamentos em farmácias particulares (11).

O total das prescrições para os medicamentos encontrados é menor do que os resultados apresentados em Uganda (31) e maior do que os resultados apresentados por estudos prévios no Brasil $(7,8,12)$ e no exterior $(6)$. Esse maior número de prescrições para os medicamentos encontrados coaduna com a maior procura para adquiri-los no Centro de Saúde. Entretanto, a quantidade de unidades/doses estocadas surpreende. A maior quantidade de medicamentos estocados era daqueles que tinham a prescrição médica (7).

A média de medicamentos por paciente também é preocupante, podendo sugerir o fenômeno da polifarmácia entre este grupo. Quando comparada a estudo realizado no Brasil por Schenkel e cols (2005) e no Irã por Zargarzadeh e cols (2005), a média de medicamentos do presente estudo é inferior. Esta média é semelhante ao valor encontrado em Passo Fundo, Rio Grande do Sul (8) e, finalmente, superior aos resultados dos demais estudos pesquisados no Brasil (10, 14, 27).

Sabe-se que a população vem adquirindo quantidades de medicamentos maiores em relação ao que realmente precisa. Como consequência, tem-se um aumento do número de medicamentos fora de uso ou impróprios para o consumo. O consumo destes medicamentos implica em sérios perigos à saúde (7).

A quantidade elevada de medicamentos adequados para uso e que são armazenados em casa pode sugerir que os pacientes não aderem ao tratamento $(13,14)$; ou que talvez não tenha sido respeitada a conduta de dispensação fracionada de medicamentos, apesar da existência da Resolução da Diretoria Colegiada (RDC) n n 80 de 2006 da Anvisa/Ministério da Saúde, que trata especificamente do fracionamento de medicamentos, de modo a fornecer quantidades individualizadas para atender às necessidades terapêuticas dos usuários (33).

Também existe a hipótese de haver acontecido troca de medicamentos e o usuário continuar com a guarda dos medicamentos anteriores (6); ou então, que o usuário tenha sido atendido por vários profissionais médicos/ dentistas num mesmo período de tempo, somando as prescrições (31). Por isso é fundamental a implantação da estratégia de Conciliação Medicamentosa. Nesta ação, é realizada uma listagem completa dos medicamentos que o paciente utiliza habitualmente, que é comparada com a prescrição de medicamentos em todos os níveis do cuidado hospitalar (admissão, alta ou transferência entre setores de internação). Esta abordagem pre- vine erros de medicação resultantes de informações incompletas ou erradas em pontos de transição no processo de atendimento ao paciente (35).

A quantidade encontrada de medicamentos não apropriados para o consumo é preocupante, sendo muito semelhante à encontrada por outros estudos no Brasil (7, $10,11)$ e no exterior $(6,31)$. Este é um problema que preocupa e que pode comprometer, ao longo dos anos, a política pública de distribuição de medicamentos e a economia familiar, quando da aquisição dos medicamentos pelo próprio paciente.

Problemas relacionados à forma de armazenamento dos medicamentos também estão de acordo com a literatura consultada $(6,11,12,31)$ e mostram que este problema é de difícil solução. Comumente, o quarto foi o local preferido para a estocagem dos medicamentos (10, $11)$, diferentemente da cozinha, local mais frequente encontrado em outros estudos $(7,12)$.

A prevalência de medicamentos para o sistema nervoso, seguidos pelos medicamentos para o sistema cardiovascular está de acordo com o encontrado por outros estudos no Brasil (11). Há uma maior quantidade de unidades/doses de medicamentos para tratamento de sistema cardiovascular mostrando que o Acidente Vásculo-Encefálico é o maior responsável pela restrição domiciliar e a Hipertensão Arterial Sistêmica como comorbidade mais frequente (34).

A diferença entre este trabalho da maior parte dos estudos anteriores sobre estoque domiciliar de medicamentos que deve ser aqui ressaltada é o grande número de medicamentos de uso contínuo apresentada pelo paciente com restrição domiciliar. Os pacientes que compõem a presente amostra são portadores de doenças crônico-degenerativas e suas sequelas. A Prefeitura Municipal de Belo Horizonte em suas Unidades Básicas de Saúde libera, por prescrição de medicamentos para uso contínuo, o equivalente de medicamentos para ser consumida em 30 dias (36).

Compreende-se que este estudo apresenta limitações. Os seus resultados não podem ser generalizados para toda população, pois são fortemente determinados por um grupo de indivíduos cujas características são as idades avançadas, as doenças crônico-degenerativas e suas consequências e o seu baixo poder econômico e de escolaridade. Por outro lado, os achados aqui podem traçar um perfil de um grupo populacional altamente dependente dos serviços de saúde, para as quais a efetividade e a eficiência dos serviços públicos de saúde podem significar uma melhor qualidade de vida. 
São recomendadas ações no sentido de uma atuação mais participativa dos Agentes Comunitários de Saúde e das Equipes da Estratégia de Saúde da Família junto aos usuários e seus familiares, como corresponsável na coleta e/ou descarte de medicamentos inapropriados e/ou que não esteja mais em uso, ou mesmo que possam ser dispensados a outras pessoas necessitadas.

\section{CONCLUSÃO}

O acesso ao medicamento via Sistema Único de Saúde é um fato para os pacientes com restrição domiciliar da área de abrangência do Centro de Saúde (CS) Jaqueline I, regional Norte, Município de Belo Horizonte.
A dispensação farmacêutica ocorre perante a apresentação da prescrição médico-odontológica e a maioria dos medicamentos encontrados nos domicílios de pacientes com movimentação restrita são medicamentos essenciais.

A quantidade de medicamentos encontrada e armazenada é excessiva, provavelmente sendo um indicador que talvez o usuário não esteja seguindo o regime posológico proposto pelo médico ou dentista, ou então que a dispensação fracionada dos medicamentos não tem sido realizada corretamente.

Pode ser sugerido, por fim, que seja trabalhado, na comunidade, o conceito de Revisão de Utilização de Medicamentos, como uma estratégia para o uso racional de medicamentos.

\section{REFERÊNCIAS}

1. Wondimu A, Molla F, Demeke B, Eticha T, Assen A, Abrha S, Melkam W. Household Storage of Medicines and Associated Factors in Tigray Region, Northern Ethiopia. Plos One. 2015; 10(8): e0135650. DOI: 10.1371/journal.pone. 0135650

2. Ocan M, Bbosa G, Waako P, Ogwal-Okeng J, Obua C. Factors predicting home storage of medicines in Northern Uganda. BMC Public Health. 2014; 14:650-656. DOI: 10.1186/1471-2458-14-650.

3. Yousif MA. In-home drug storage and utilization habits: a Sudanese study. East Mediterr Health J. 2002; 8 (2/3): 422-431.

4. Stratchounski LS, Andreeva IV, Ratchina S A, Galkin DV, Petrotchenkova N A, Demin A A, Kuzin VB, Kusnetsova ST, Likhatcheva RY, Nedogoda SV, Ortenberg EA, Belikov AS, Toropova IA. The Inventory of Antibiotics in Russian Home Medicine Cabinets. Clin Infect Dis. 2003; 37(4):498-505.

5. Aljinoviæ-Vuèiæ V, Trkulja V, Lackoviæ Z. Content of Home Pharmacies and Self-Medication Practices in Households of Pharmacy and Medical Students in Zagreb, Croatia: Findings in 2001 with a Reference to 1977. Croat Med J. 2005; 46 (1):74-80

6. Jassim AB. In-home Drug Storage and Self-medication with Antimicrobial Drugs in Basrah, Iraq. Oman Med J. 2010; 25 (2):79-86. DOI: 10.5001/omj.2010.25.

7. Schenkel EP, Fernandes LC, Mengue SS. Como São Armazenados os Medicamentos Nos Domicílios? Acta Farm. Bonaerense. 2005; 24(2): 266-270.

8. Dal Pizzol TS, Piccoli A, Brugnera, Q, Schenkel EP, Mengue SS. Análise dos Estoques Domiciliares de Medicamentos Essenciais no Sul do Brasil. Acta Farm. Bonaerense. 2006; 25 (4):601-607.

9. Ferreira WA, Silva MEST, Paula ACCFF, Resende CAMB. Avaliação de farmácia caseira no município de Divinópolis (MG) por estudantes do Curso de farmácia da Unifenas. Infarma. 2005; 17(7/9):84-86.

10. Tourinho FSV, Bucharetchi F, Sthephan C, Cordeiro R. Children, adolescents, home pharmacies, home medicine chests, drugs, self-medication, pharmacoepidemiology. J Pediatr. 2008; 84 (5):416-422. DOI:10.2223/JPED.1831

11. Ribeiro MA, Heinecke I. Estoque Domiciliar de Medicamentos na Comunidade Ibiaense Acompanhada pelo Programa Saúde da Família, em Ibiá-MG, Brasil. Saúde Soc. 2010; 19(3): 653-663.

12. Laste G, Deitos A, Kauffmann C, Castro LC, Torres ILS, Fernandes LC. Papel do agente comunitário de saúde no controle do estoque domiciliar de medicamentos em comunidades atendidas pela estratégia de saúde da família. Cien Saude Colet. 2012; 17(5):1305-1312. DOI: 10.1590/ S1413-81232012000500024

13. Brum CA, Depizzol MCA, Lopes TV, Loures GF, Valadão AF. Avaliação do estoque de medicamentos das residências da Região do Vale do Aço-MG. Rev. Bras. Farm. 2007; 88(4):173-176.

14. Beckhauser GC, Valgas C, Galato D. Perfil do estoque domiciliar de medicamentos em residências com crianças. Rev Ciênc Farm Básica Apl. 2012; 33 (4):583-589.

15. Bortolon PC, Medeiros EFF, Naves JOS, Karnikowski MGO, Nóbrega OT. Análise do perfil de automedicação em mulheres idosas brasileiras. Cien Saude Colet. 2008; 13 (4): 1219-1226. DOI: 10.1590/S141381232008000400018

16. Marin MJS, Cecílio LCO, Perez AEWUF, Santella F, Silva CBA, Gonçalves Filho JR, Roceti LC. Caracterização do uso de medicamentos entre idosos de uma unidade do 
Programa Saúde da Família. Cad. Saúde Pública. 2008; 24 (7):1545-1555.

17. Ursine PGS, Cordeiro HÁ, Moraes CL. Prevalência de idosos restritos ao domicilio em região metropolitana de Belo Horizonte (Minas Gerais, Brasil). Cien Saude Colet. 2011;16 (6): 2953-2962. DOI: 10.1590/S141381232011000600033

18. BRASIL, Ministério da Saúde, SAGE- Sala de Apoio a Gestão Estratégica. Disponível em http://sage.saude.gov. br . [Acesso em 24/02/2016]

19. Lima GB, Nunes LCC, Barros JAC. Uso de medicamentos armazenados em domicílio em uma população atendida pelo Programa Saúde da Família. Cien Saude Colet. 2010; 15 (3):3517-3522. DOI: 10.1590/S141381232010000900026

20. BRASIL. Ministério da Saúde, Secretaria de Ciência, Tecnologia e Insumos Estratégicos. Departamento de Assistência Farmacêutica e Insumos Estratégicos Relação de Medicamentos Essenciais, RENAME 2014, $9^{\mathrm{a}}$ ed.

21. WHO. World Health Organization. Guidelines for ATC classification and DDD assignment 2013, Oslo, Norway, December 2012，16 $6^{\text {th }}$ edition, 2013. Disponível em: http://www.whocc.no/filearchive/publications/1_ 2013guidelines.pdf [Acessado em 21/08/2015]

22. BRASIL. Ministério do Planejamento, Orçamento e Gestão. IBGE. Perfil dos idosos responsáveis pelos domicílios, 2002. Disponível em: http://www.ibge.gov.br/home/ presidencia/noticias/25072002pidoso.shtm [Acessado em 03/08/2015]

23. Marques GQ, Freitas IBA. Experiência-piloto de assistência domiciliar: idosos acamados de uma unidade básica de saúde, Porto Alegre, Brasil. Rev Esc Enferm USP. 2009; 43 (4): 825-832.

24. Giacomin KC, Peixoto SV, Uchôa E, Lima-Costa MF. Estudo de base populacional dos fatores associados à incapacidade funcional entre idosos na Região Metropolitana de Belo Horizonte, Minas Gerais, Brasil. Cad. Saúde Pública. 2008; 24 (6):1260-1270.

25. Silva AF, Abreu CRO, Barbosa EMF, Raposo NRB, Chicourel EL. Problemas relacionados aos medicamentos em idosos fragilizados da Zona da Mata Mineira, Brasil. Rev. Bras. Geriatr. Gerontol. 2013; 16 (4):691-704.

26. Santos DM, Sousa SNS, Silva DRS, Silva JC, Figueiredo MLF. Regime terapêutico inadequado em idosos acamados no domicílio. REME Rev. Min. Enferm. 2009;13 (2):177-182.

27. Lacerda SM, Gazzola JM, Lopes AB, Lemos NFD, Cordeiro RC. Qualidade de vida de idosos atendidos em Pro- grama de Assistência Domiciliária. Rev. Bras. Geriatr. Gerontol. 2011; 14 (2):329-342.

28. Veras RP, Ramos LR, Kalache, A. Crescimento da população idosa no Brasil: Transformações e consequências na sociedade. Rev. Saúde Públ. 1987; 21 (3): 225-233.

29. Freedman VA, Wolf DA, Spillman BC. Disability-Free Life Expectancy Over 30 Years: A Growing Female Disadvantage in the US Population. Am J Public Health. 2016; 106:1079-1085. DOI: 10.2105/AJPH.2016.303089

30. BRASIL. Agência Nacional de Vigilância Sanitária (Brasil). Resolução $\mathrm{RDC} \mathrm{n}^{\circ} 20$, de 5 de maio de 2011. Dispõe sobre o controle de medicamentos à base de substâncias classificadas como antimicrobianos, de uso sob prescrição, isoladas ou em associação. Diário Oficial da República Federativa do Brasil, Poder Executivo, Brasília, DF, 9/05/2010, SEÇÃO 2, PÁGINA 39. Disponível em: http://www.jusbrasil.com.br/diarios/26573265/dou-secao-1-09-05-2011-pg-39/pdfView [Acessado em: $1 / 06 / 2012]$.

31. Kalyango JN, Hall M, Karamagi C. Home medication management practices and associated factors among patients with selected chronic diseases in a community pharmacy in Uganda. BMC Health Serv Res. 2012;12:323-331. DOI: 10.1186/1472-6963-12-323.

32. Zargarzadeh AH, Tavakoli N, Hassanzadeh A. A survey on the extent of medication storage and wastage in urban Iranian households. Clin Ther. 2005; 27(6):972-978. DOI: 10.1016/S0149- 2918(05)00122-0

33. BRASIL. Ministério da Saúde, Agência Nacional de Vigilância Sanitária. Resolução da Diretoria ColegiadaRDC N.80 de 11 de maio de 2006. Dispõe sob o fracionamento de medicamentos. Brasília, 11 maio 2006. [Acessado em 31/08/2015]

34. Flores LM, Mengue SS. Uso de medicamentos por idosos em região do Sul do Brasil. Rev.Saúde Pública. 2005; 39 (6):924-929.

35. WHO. World Health Organization. High 5s: Action on Patient Safety Standard Operating Protocol Fact Sheet: Medication Reconciliation.2012. Disponível em: http:// www.who.int/patientsafety/implementation/solutions/high5s/ps_med_rec_fs_... [Acessado em 31/07/2017]

36. SMS-BH. Belo Horizonte, Secretaria Municipal de Saúde, Portaria SM AS/SUS- BH, n ${ }^{\circ}$ 038/98, 03 de dezembro de 1998. Diário Oficial do Município. Ano IV, Edição 780. Disponível em: file:///D:/Documento/gilberto/ DOM\%20-\%20Di\%C3\%A1 rio\%20Oficial\%20do\%20 Munic\%C3\%ADpio\%20_\%20portaria\%20309\%20dias. html. [Acessado em 31/07/2017] 